\title{
ASTHMA
}

\section{Effect of two breathing exercises (Buteyko and pranayama) in asthma: a randomised controlled trial}

\section{S Cooper, J Oborne, S Newton, V Harrison, J Thompson Coon, S Lewis, A Tattersfield}

See end of article for authors' affiliations

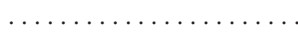

Correspondence to: Ms S Cooper, Division of Respiratory Medicine, Clinical Sciences Building, City Hospital, Nottingham NG5 1PB, UK: sue.cooper@ nottingham.ac.uk

Revised version received 10 April 2003

Accepted for publication 24 April 2003
Background: Patients with asthma are interested in the use of breathing exercises but their role is uncertain. The effects of the Buteyko breathing technique, a device which mimics pranayama la yoga breathing technique), and a dummy pranayama device on bronchial responsiveness and symptoms were compared over 6 months in a parallel group study.

Methods: Ninety patients with asthma taking an inhaled corticosteroid were randomised after a 2 week run in period to Eucapnic Buteyko breathing, use of a Pink City Lung Exerciser (PCLE) to mimic pranayama, or a PCLE placebo device. Subjects practised the techniques at home twice daily for 6 months followed by an optional steroid reduction phase. Primary outcome measures were symptom scores and change in the dose of methacholine provoking a $20 \%$ fall in $\mathrm{FEV}_{1}\left(\mathrm{PD}_{20}\right)$ during the first 6 months.

Results: Sixty nine patients $(78 \%)$ completed the study. There was no significant difference in $\mathrm{PD}_{20}$ between the three groups at 3 or 6 months. Symptoms remained relatively stable in the PCLE and placebo groups but were reduced in the Buteyko group. Median change in symptom scores at 6 months was 0 (interquartile range -1 to 1 ) in the placebo group, $-1(-2$ to 0.75$)$ in the PCLE group, and -3 $(-4$ to 0$)$ in the Buteyko group ( $p=0.003$ for difference between groups). Bronchodilator use was reduced in the Buteyko group by two puffs/day at 6 months; there was no change in the other two groups $(p=0.005)$. No difference was seen between the groups in $\mathrm{FEV}_{1}$, exacerbations, or ability to reduce inhaled corticosteroids.

Conclusion: The Buteyko breathing technique can improve symptoms and reduce bronchodilator use but does not appear to change bronchial responsiveness or lung function in patients with asthma. No benefit was shown for the Pink City Lung Exerciser.
$\mathrm{T}$ here is interest in the use of complementary treatment for asthma and a third of respondents in a recent asthma survey had tried one or more breathing techniques to relieve symptoms. ${ }^{1}$ However, few controlled studies have been undertaken to determine the effectiveness of these approaches. We have previously studied two components of pranayama, ${ }^{2}$ the yoga breathing exercise, by asking subjects with asthma to breathe twice a day through a cylinder with an expiratory resistance (the Pink City Lung Exerciser (PCLE)) designed to reduce breathing frequency and increase the duration of expiration. There was a small reduction in airway responsiveness to histamine after using the PCLE for 2 weeks compared with a placebo device without a resistance.

A BBC television programme in 1998 generated considerable interest in the Buteyko breathing technique. The technique, developed in Russia by Konstantin Buteyko, is based on the belief that asthma is caused by hyperventilation and hypocapnia and can be cured in most patients by using special techniques to reduce minute ventilation. ${ }^{3}$ The effects on asthma control in two small controlled studies in Australia were modest, however. ${ }^{45}$ The Eucapnic Buteyko method is an adaptation of the Russian technique which was first introduced in Australia but is now used worldwide. It includes the same focus on ventilation control but the approach has been designed to suit patients in western countries.

We compared the effect of the Buteyko technique, pranayama exercises using the PCLE, and the PCLE placebo device in subjects with asthma. Symptom scores and bronchial responsiveness to methacholine were the primary outcome measures.

\section{METHODS}

\section{Subjects}

Non-smoking volunteers aged 18-70 with stable asthma but no other important illness were recruited between September
1999 and November 2000 from our asthma volunteer database and from posters and newspaper advertisements. Inclusion criteria included: taking an inhaled short acting $\beta$, agonist at least twice a week and regular inhaled corticosteroids with no change in dose in the preceding 4 weeks; pre-bronchodilator forced expiratory volume in 1 second $\left(\mathrm{FEV}_{1}\right)$ of at least $50 \%$ predicted and a 10\% increase following $400 \mu \mathrm{g}$ inhaled salbutamol; a provocative dose of methacholine causing a $20 \%$ fall in $\mathrm{FEV}_{1}\left(\mathrm{PD}_{20}\right)$ of $10.24 \mu \mathrm{mol}$ or less; and a mean daily symptom score of one or more during the run in period. Subjects taking treatment other than sodium cromoglycate (one subject) were excluded.

Written consent was obtained from all subjects and Nottingham City Hospital ethics committee approved the study.

\section{Measurements}

$\mathrm{FEV}_{1}$ and forced vital capacity (FVC) were measured with a dry bellows spirometer (Vitalograph, Buckingham, UK) as the highest of two successive readings within $100 \mathrm{ml}$. Methacholine $\mathrm{PD}_{20}$ was measured by a breath activated dosimeter (MEFAR, Brescia, Italy). Nebulisers had an output of 10 $(0.5) \mu \mathrm{l}$ at 1 second and calibration was checked every 6 months. Subjects inhaled three puffs of saline followed by doubling doses of methacholine (dose range 0.02-42 $\mu \mathrm{mol}$ ) until $\mathrm{FEV}_{1}$ had fallen by $20 \%$ from the post-saline value. FEV was measured 2 minutes after each dose and $\mathrm{PD}_{20}$ by linear interpolation on a log-dose response plot.

Subjects kept diary cards to record breathing exercises, medication use, and asthma symptom severity during the day and night (both $0-5$ ), plus activity limitation due to asthma (0-3; maximum combined score 13). Quality of life was assessed using the SF-36 ${ }^{6}$ and Asthma Quality of Life $^{7}$ questionnaires. 


\section{Protocol}

The study had a parallel group design with participants randomised to be taught the Eucapnic Buteyko technique or use of the PCLE or PCLE placebo device. Subjects underwent training (see below) and were asked to perform the exercises for at least 15 minutes twice a day throughout the study. A card sent 2 weeks later gave further encouragement. Subjects were followed for 6 months with an optional extension during which inhaled steroid reduction was attempted.

Bronchodilator reversibility and methacholine $\mathrm{PD}_{20}$ were measured on separate screening visits. Subjects fulfilling the entry criteria entered a 2 week run in period. Eligible subjects were then allocated to one of the three treatment groups using the next available number from computer generated numbers, randomised in blocks of six, and using sealed envelopes prepared independently. To ensure sufficient numbers for the Buteyko sessions, randomisation was skewed towards the Buteyko group except for the last 12 patients who were randomised between the two PCLE groups. Subjects were only given details of their treatment. They attended the clinic after $1,3,5,9,13$, and 26 weeks at the same time of day after withholding $\beta_{2}$ agonists for 6 hours. Subjects were seen first by the training coordinator who dealt with queries about the breathing exercises. They then saw the clinical assessor who assessed asthma control and measured lung function, including methacholine $\mathrm{PD}_{20}$ at 13 and 26 weeks. The assessor was not told which breathing technique subjects were using and subjects were asked not to mention it. Subjects completed the two quality of life questionnaires before randomisation and after 13 and 26 weeks, together with a postal feedback questionnaire at least 6 weeks after completing the study.

Subjects were asked to keep their dose of inhaled steroid constant throughout the first 6 months unless they had an asthma exacerbation. This was defined as a $20 \%$ fall in $\mathrm{FEV}_{1}$ or a $50 \%$ increase in $\beta_{2}$ agonist use and $>6$ puffs on two of three successive days compared with the randomisation visit/run in period, or worsening asthma needing urgent medical care or rescue medication on two of three successive nights.

\section{Steroid reduction phase}

Steroid reduction was attempted 6 months after randomisation for subjects with stable asthma who agreed to take part. The dose was reduced by $25 \%$ every 3 weeks at a clinic visit until either the inhaled steroid had been discontinued, an asthma exacerbation occurred, or the doctor or subject felt that asthma control had deteriorated to an extent that merited reversal of steroid reduction.

\section{Training}

Training was given in small groups: numbers ranged from 3-8 in the Buteyko training sessions, $2-5$ in the PCLE group, and $1-5$ in the control group.

\section{Buteyko group}

The Eucapnic Buteyko technique, a western modification of the Russian Buteyko technique, was taught by a certified practitioner in five 2 hour sessions over a weekend or successive evenings. Subjects were taught exercises to reduce the frequency and depth of breathing and, as part of the technique, were asked to record pulse and breath holding time twice daily in a diary. They were instructed to use the breathing technique twice daily and to relieve asthma symptoms, and only to use their bronchodilator if that failed. Subjects were also asked to tape their mouth at night with hypoallergenic tape (Micropore) to prevent mouth breathing and were advised to avoid factors such as stress, exercise accompanied by deep breathing, oversleeping, and certain foods (for example, highly processed food and additives). Two weeks after the training the subjects were telephoned by the Buteyko practitioner to give encouragement and answer que- ries. Subjects could contact the practitioner for further advice if they felt it necessary.

\section{Pink City Lung Exerciser (PCLE) group}

Training took place in a single session. The PCLE consists of a mouthpiece attached to a disc containing six apertures 2-5 $\mathrm{mm}$ in diameter with one-way valves which halve the cross sectional area during expiration and thus impose a $1: 2$ ratio on the duration of inspiration compared with expiration. $^{2}$ With the device set to the largest aperture, subjects were asked to breathe in and out from residual volume to total lung capacity at a rate at which they felt no resistance to breathing and could feel no cheek movement. If, after a week, they could do the exercises without feeling breathless, they were encouraged to decrease the aperture size gradually to reduce respiratory rate. They were told to use their $\beta_{2}$ agonist only for relief of symptoms.

\section{Control group}

Training was identical to that for the PCLE group. The placebo device had an identical appearance to the PCLE but the apertures had no valve and a leak ensured no resistance to breathing.

\section{Outcome measures}

The primary efficacy variables were symptom scores and methacholine $\mathrm{PD}_{20}$. Secondary outcomes included $\mathrm{FEV}_{1}$, bronchodilator use, asthma exacerbation rates, quality of life assessments, and reduction in inhaled corticosteroid dose.

With 30 subjects in each group there was $90 \%$ power to detect a difference between treatments in change from baseline methacholine $\mathrm{PD}_{20}$ of 1.5 doubling doses using an $\alpha$ of 0.05 according to previous data. ${ }^{2}$

\section{Analysis of data}

All available data for the 89 patients who underwent training were analysed. When data were unavailable they were treated as missing. Codes were revealed after all data had been reviewed and entered. $\mathrm{PD}_{20}$ values were log transformed for analysis and expressed as change from the end of the run in period, as were $\mathrm{FEV}_{1}$, symptoms, and quality of life measures. Change in $\mathrm{PD}_{20}$ and $\mathrm{FEV}_{1}$ were compared between groups using analysis of variance, adjusting for baseline values as a covariate when there were baseline differences. The KruskalWallis test was used to compare changes from baseline for steroid reduction, quality of life measures, median symptom scores, and bronchodilator use (for 7 days before each visit). We also carried out an analysis of the primary outcome measures, substituting missing data with the last recorded value before subjects withdrew. The results were virtually identical to those seen in our main analysis and are not presented.

\section{RESULTS}

Of 178 patients screened, 136 fulfilled the entry criteria, 90 were randomised, 89 underwent training, and 69 completed the 6 month study (fig 1). Data from one subject who became pregnant were excluded from the analysis before breaking the randomisation code. The number of participants failing to complete and the reasons given were similar in the three groups. Baseline characteristics were also similar except that those receiving the Buteyko training were younger, were taking slightly more inhaled corticosteroid, and had a lower $\mathrm{PD}_{20}$ methacholine (table 1). (Baseline quality of life data (table $\mathrm{lb}$ ) are available online at www.thoraxjnl.com/ supplemental.)

\section{Primary outcome measures}

There was little change in $\mathrm{PD}_{20}$ from baseline during the study and no significant difference between the groups at 3 and 6 months (table 2). 


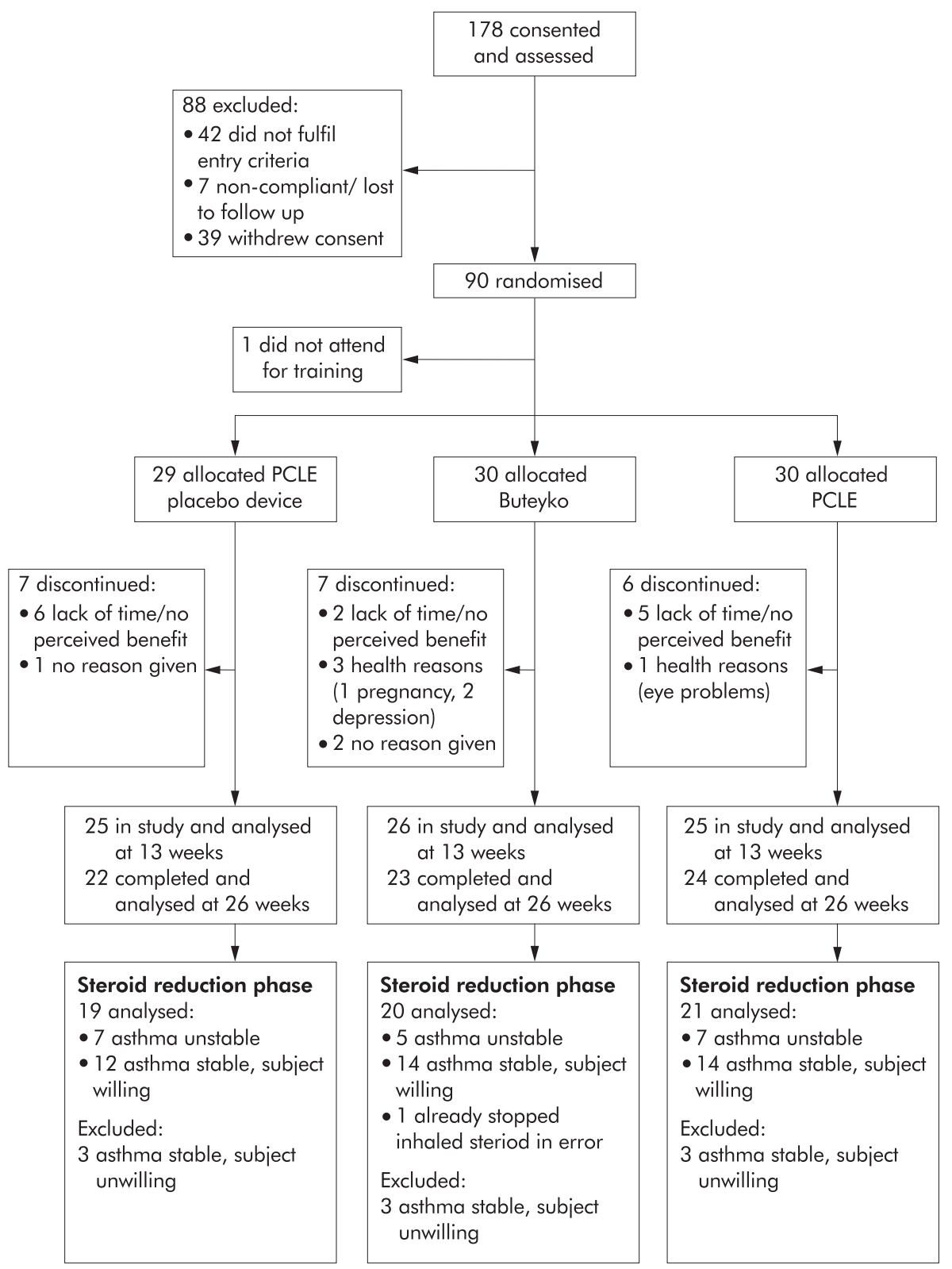

Figure 1 Summary of patients entered, randomised and withdrawn from trial.

Symptoms remained relatively stable in the PCLE and placebo groups but were reduced in the Buteyko group (fig 2). The median (interquartile range, IQR) change in daily symptom scores at 6 months was $0(-1$ to 1$)$ in the placebo group, -1 ( -2 to 0.75 ) in the PCLE group, and -3 ( -4 to 0$)$ in the Buteyko group; the difference between the three groups was significant $(\mathrm{p}=0.003)$.

\section{Secondary outcome measures}

The use of $\beta_{2}$ agonists was reduced in the Buteyko group throughout the study and unchanged in the other two groups: median (IQR) change at 6 months 0 ( -2 to 0$)$ puffs/day in the placebo group, 0 ( -2 to 0 ) puffs/day in the PCLE group, and -2 $(-4$ to 0$)$ puffs/day in the Buteyko group (difference between groups significant, $\mathrm{p}=0.005)$. There was no difference in change in $\mathrm{FEV}_{1}$, number of asthma exacerbations, days requiring an increase in inhaled corticosteroid dose or courses of prednisolone between the groups during the 6 month study period (table 3 ). Changes in quality of life, as measured by the SF-36 questionnaire, did not differ significantly apart from one of the eight dimensions ("Role limitation due to physical problems") which improved more in the Buteyko group at both 3 and 6 months (both $\mathrm{p}<0.01$ ). None of the Asthma Quality of Life questionnaire domains had changed significantly at 6 months (see table 4 online at www.thoraxjnl.com/ supplemental for details)

\section{Steroid reduction phase}

Of 69 subjects who completed the study, nine were unwilling to attempt steroid reduction leaving 60 in the analysis with similar numbers in each group (fig 1). The extent to which subjects could reduce their inhaled steroid did not differ significantly between groups ( $p=0.7$; table 3 , fig 3 )

\section{Feedback from subjects}

At the end of the study more subjects in the Buteyko group thought that they had definitely or probably received active treatment $(15+8$ of 26 respondents) than subjects in the PCLE group $(4+9$ of 25$)$ or the placebo PCLE group $(4+10$ of 25) $(\mathrm{p}<0.001)$.

There were 72 replies (81\% response rate) to the postal questionnaire. Most subjects said they had been compliant 
Table 1 Baseline characteristics of study patients

\begin{tabular}{|c|c|c|c|}
\hline & $\begin{array}{l}\text { Control } \\
(n=29)\end{array}$ & $\begin{array}{l}\text { Buteyko } \\
(n=30)\end{array}$ & $\begin{array}{l}\text { PCLE } \\
(n=30)\end{array}$ \\
\hline Age (years) & $47(11)$ & $40(11)$ & $45(13)$ \\
\hline $\mathrm{M} / \mathrm{F}$ & $18 / 11$ & $15 / 15$ & $16 / 14$ \\
\hline Inhaled corticosteroid dose ( $\mu \mathrm{g})$ & $603(330)$ & $757(370)$ & $608(529)$ \\
\hline Never/ex-smokers & $16 / 13$ & $20 / 10$ & $15 / 15$ \\
\hline Duration of asthma (years) & $23(15.3)$ & $24(14.7)$ & $23(14.1)$ \\
\hline FEV (I) & $2.71(0.89)$ & $2.58(0.76)$ & $2.64(0.94)$ \\
\hline $\mathrm{FEV}_{1}(\%$ predicted $)$ & $82(21.1)$ & $77(16.4)$ & $80(17.6)$ \\
\hline Reversibility (\%) & $17(8.3)$ & $19(13.3)$ & $16(6.2)$ \\
\hline Median (IQR) $\beta_{2}$ agonist use (puffs/day) & $2(0-3.8)$ & $2(0-4)$ & $2(0-4)$ \\
\hline Geometric mean (IQR) $\mathrm{PD}_{20}$ methacholine (umol) & $0.66(0.2-2.1)$ & $0.19(0.07-0.6)$ & $0.65(0.2-2.8)$ \\
\hline Median (IQR) symptom score & $3.5(2-5.8)$ & $4(2.8-5.3)$ & $4(3-6)$ \\
\hline Overall asthma quality of life score & $5.0(0.8)$ & $5.1(1.0)$ & $4.9(0.8)$ \\
\hline
\end{tabular}

Values are mean (SD) unless stated otherwise.

$\mathrm{PCLE}=$ Pink City Lung Exerciser; $\mathrm{FEV}_{1}=$ forced expiratory volume in 1 second; $\mathrm{PD}_{20}=$ dose of methacholine provoking a fall in $\mathrm{FEV}_{1}$ of $20 \%$ or more.

${ }^{\star}$ More detailed quality of life baseline measurements are available online in table $1 \mathrm{~b}$ at www.thoraxjnl.com/ supplemental.

\begin{tabular}{|c|c|c|c|c|c|}
\hline & \multirow[b]{2}{*}{ Control } & \multirow[b]{2}{*}{ Buteyko } & \multirow[b]{2}{*}{ PCLE } & \multicolumn{2}{|c|}{ Estimated difference* $(95 \% \mathrm{Cl})$ between: } \\
\hline & & & & Buteyko $v$ control & PCLE $v$ control \\
\hline \multicolumn{6}{|l|}{13 weeks } \\
\hline$n$ & 25 & 26 & 24 & & \\
\hline Mean & 0.25 & 0.28 & 0.12 & $-0.3(-1.2$ to 0.5$)$ & $-0.03(-0.8$ to 0.8$)$ \\
\hline SE & 0.28 & 0.33 & 0.27 & & \\
\hline \multicolumn{6}{|l|}{26 weeks } \\
\hline & 22 & 22 & 23 & & \\
\hline Mean & -0.04 & 0.66 & 0.44 & $0.1 \quad(-1.0$ to 1.2$)$ & $0.6(-0.4$ to 1.6$)$ \\
\hline SE & 0.39 & 0.26 & 0.44 & & \\
\hline
\end{tabular}

$\mathrm{PCLE}=$ Pink City Lung Exerciser

$\mathrm{PD}_{20}$ is measured in doubling doses. Two subjects who completed the study did not have a $\mathrm{PD}_{20}$ measurement at 6 months.

${ }^{*}$ Adjusted for baseline values.

with the breathing exercises during the study (see table 5 online at www.thoraxjnl.com/supplemental). Of 23 responders in the Buteyko group, 22 said they had practised the exercises twice a day while 14 in the PCLE group and 17 in the placebo PCLE group said they had practised for at least 10 minutes a day. Four subjects in the Buteyko group were unable to tape their mouth at night but 14 said they did it for at least five nights a week and eight did it every night. After completing the study 16 subjects in the Buteyko group had continued the exercises, at least on an occasional basis, compared with 12 and 9 in the PCLE and placebo PCLE groups.

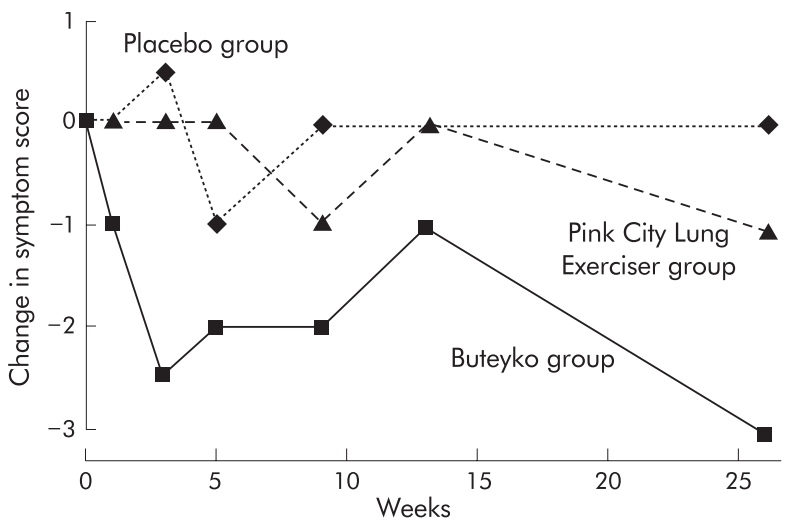

Figure 2 Median change in symptom scores during the 6 month study.

\section{DISCUSSION}

In this randomised controlled trial subjects taught the Eucapnic Buteyko technique had reduced asthma symptoms and bronchodilator use compared with the other two groups but there was no difference in bronchial responsiveness or $\mathrm{FEV}_{1}$. No difference was seen between the PCLE and PCLE placebo device for any outcome measure.

A television programme in 1998 in the UK generated considerable media and patient interest in the Buteyko technique. The course used in this study, the Eucapnic Buteyko technique, was available to the general public. It includes breathing exercises, taping the mouth at night, strongly discouraging the use of $\beta_{2}$ agonists except in emergencies, advice on lifestyle, and strong practitioner encouragement. Yoga breathing exercises (pranayama) involves mental concentration to cause a reduction in breathing frequency, a $1: 2$ ratio of inspiration to expiration, and a pause at the end of inspiration and expiration. The expiratory resistance in the PCLE imposes a reduction in breathing frequency and expiratory flow.

Over $90 \%$ of 100000 patients who completed the Buteyko course in Russia are said to need no further asthma medication and a similar success rate has been claimed for 8000 patients in Australia.' The results of the two controlled studies from Australia in 39 and 36 subjects are more modest. ${ }^{45}$ In one, which focused on patients taking high doses of short acting $\beta_{2}$ agonists (median $>800 \mu \mathrm{g}$ salbutamol daily), Buteyko training resulted in a large reduction in $\beta_{2}$ agonist use which correlated with a reduction in minute ventilation compared with the control group. There was a 
Table 3 Secondary end points over the 6 month study period

\begin{tabular}{|c|c|c|c|c|}
\hline & Control & Buteyko & PCLE & $\mathrm{p}$ value* \\
\hline Mean (SD) change in $\mathrm{FEV}_{1}(\mathrm{I})$ over 26 weeks & $0.001(0.14)$ & $0.06(0.26)$ & $-0.002(0.14)$ & 0.4 \\
\hline Change in $\beta_{2}$ agonist use (puffs/day) at 26 weeks & $0(-2$ to 0$)$ & $-2(-4$ to 0$)$ & $0(-2$ to 0$)$ & 0.005 \\
\hline Number of exacerbations & $1(0-2)$ & $1(0-1.75)$ & $1(0-2)$ & 0.7 \\
\hline Days taking increased dose of inhaled steroid & $0(0-16)$ & $0(0-11)$ & $1(0-7)$ & 0.9 \\
\hline Median (range) number of prednisolone courses per subject & 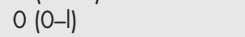 & $0(0-2)$ & $0(0-1)$ & 0.4 \\
\hline Change in overall asthma quality of life score $\dagger$ & $0.61(-0.11$ to 0.95$)$ & $1.03(0.19$ to 1.69$)$ & $0.57(0.07$ to 1.10$)$ & 0.2 \\
\hline Reduction in inhaled steroids $(\%)$ in steroid reduction phase & $0(0-50)$ & $41.5(0-100)$ & $25(0-62.5)$ & 0.7 \\
\hline
\end{tabular}

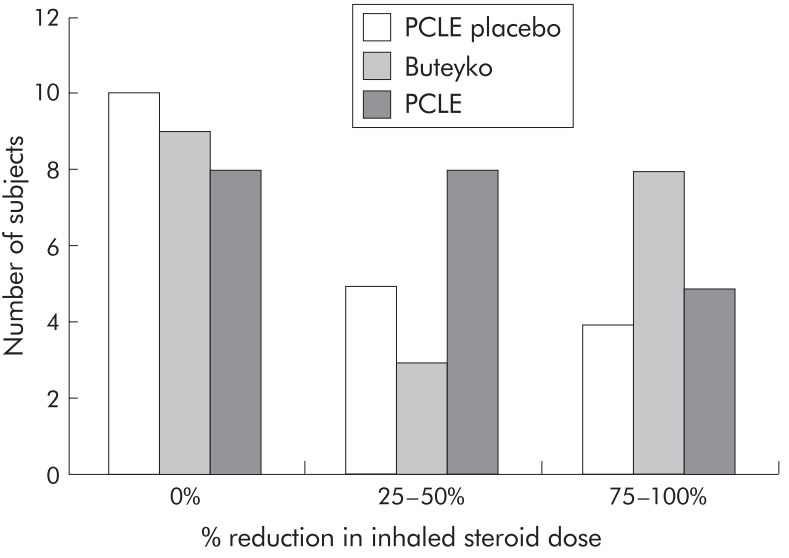

Figure 3 Number of subjects able to reduce inhaled corticosteroids in the steroid reduction phase.

non-significant trend to reduced inhaled steroid use and better quality of life, but no change in lung function or end tidal $\mathrm{CO}_{2}$ pressure. In the second study subjects taught Buteyko breathing exercises by video had a reduction in bronchodilator use and improvement in quality of life but no change in peak flow rate. ${ }^{5}$

Our study was larger and longer than the previous studies, and looked for the first time at bronchial responsiveness and asthma exacerbations. Although only averaging two puffs of $\beta_{2}$ agonist a day on entering the study, subjects in the Buteyko group were able to reduce their $\beta_{2}$ agonist use. Despite this, symptoms were reduced and there was no increase in exacerbations or use of inhaled corticosteroids. There was also an improvement in one of the eight dimensions of the SF-36 questionnaire ("Role limitation due to physical problems"). The changes in asthma quality of life and reduction in inhaled corticosteroids favoured Buteyko, but the differences were small and non-significant. There was no change in bronchial responsiveness or $\mathrm{FEV}_{1}$ despite clear potential for improvement.

These findings support the suggestion ${ }^{10}$ that the Buteyko technique may help patients adapt to their asthma and feel more in control of their treatment. It does not appear to alter the underlying disease process, however. Since subjects in the Buteyko group were strongly discouraged from using $\beta_{2}$ agonists, the extent to which the reduction in $\beta_{2}$ agonist use was a cause or a consequence of a reduction in symptoms is uncertain.

Our findings should be generalisable to patients who are interested in breathing exercises. The study is not able, however, to determine which subjects benefited from the Buteyko technique. Patients with asthma who also have dysfunctional breathing ${ }^{11}$ or who take more $\beta_{2}$ agonists than they need ${ }^{13}$ may obtain most benefit, but further studies are needed to determine this.
In a previous comparison of the PCLE and placebo device in subjects with mild asthma, the active PCLE caused a small reduction in bronchial responsiveness. ${ }^{2}$ We were unable to confirm this in the current study, perhaps because of differences in the populations studied or study duration. The PCLE involves only one aspect of yoga, and a 4 month trial of Sahaja yoga in asthma $^{14}$ showed benefit over a control intervention.

The need for controlled studies of complementary medicine is widely recognised with recent endorsement from a presidential commission in the $U^{15}$ and a House of Lords Select Committee in the UK. ${ }^{16}$ Such studies are difficult to carry out, however, and our study raises several issues. The choice of a suitable control is one difficulty; subjects in the Buteyko group were more likely to believe they were receiving active treatment resulting, perhaps, in greater psychological benefit. On the other hand, use of the placebo PCLE as a control involved subjects in some change from usual practice since they were asked to focus on breathing for 30 minutes a day. Some subjects may have benefited from this since nine continued to use the device after the study ended, despite being told it was the placebo device. Any benefit from the placebo device would reduce the ability of the study to detect an effect from the other interventions. Maintaining blindness is another potential problem. Asking subjects not to mention the technique to the assessor worked reasonably well, although one mentioned Buteyko and an occasional subject mentioned a device, but which device was not clear. A formal study may also interfere with usual practice. A previous study ${ }^{4}$ was criticised because the Buteyko group received more follow up visits than the control group so we only included one follow up telephone discussion with the Buteyko practitioner. Most subjects were happy with this but nine would have liked more contact.

A further problem is that advice from complementary medicine practitioners may differ from conventional advice and a few subjects found this difficult, particularly knowing whether to use a $\beta$, agonist or the Buteyko technique to control worsening asthma. Finally, the success or failure of a treatment such as Buteyko relies heavily on the patient/ practitioner relationship. The training sessions are long and patients need to be highly motivated to commit themselves fully to the training and need to practise daily. The success rate in a formal trial might therefore be less than that seen in everyday practice, since subjects did not specifically choose the Buteyko course and, because they were not paying, they may have felt less committed to seeing it through. Furthermore, subjects who may be considered unsuitable by Buteyko practitioners were not necessarily excluded by our entry criteria.

Despite these problems, which are inherent in all studies of complementary medicine, and the differences in study design between our study and the previous controlled studies, our findings are broadly similar. They suggest that the Buteyko technique produces some benefit by reducing symptoms and use of bronchodilators and it may improve some aspects of 
quality of life. None of the studies found any improvement in airway calibre, however, and we found no effect on bronchial responsiveness or asthma exacerbations. The Buteyko technique may be worth trying in patients who are sympathetic to the ethos and are willing to commit the time required.

\section{ACKNOWLEDGEMENTS}

The study was supported by The National Asthma Campaign. The authors thank Susan Mitchell and "Lifesource" for running the Buteyko classes and Dr Virendra Singh for supplying the Pink City Lung Exercisers and placebo devices. The authors are grateful to Tim Harrison for reviewing the manuscript and for providing medical cover and advice, along with Kate Phillips, and to Marie Cooper for help with the data entry.

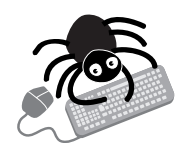

Baseline quality of life characteristics (table $1 \mathrm{~b}$ ), changes in quality of life scores (table 4), and details of the postal questionnaire sent to subjects at least 6 weeks after the end of the study (table 5) are available online at the Thorax website (www.thoraxjnl.com/supplemental).

\section{Authors' affiliations}

S Cooper, J Oborne, S Newton, V Harrison, J Thompson Coon, S Lewis, A Tattersfield, Division of Respiratory Medicine, City Hospital, Nottingham NG5 IPB, UK

\section{REFERENCES}

1 Ernst E. Complementary therapies for asthma: what patients use. J Asthma 1998;35:667-71.
2 Singh V, Wisniewski A, Britton J, et al. Effect of yoga breathing exercise (pranayama) on airway reactivity in subjects with asthma. Lancet 1990;335:1381-3.

3 Stalmatski A. Freedom from asthma: Buteyko's revolutionary treatment. London: Kyle Cathie Ltd, 1997.

4 Bowler SD, Green A, Mitchell CA. Buteyko breathing techniques in asthma: a blinded randomised controlled trial. Med J Aust 1998; 169:575-8.

5 Opat AJ, Cohen MM, Bailey M, et al. A clinical trial of the Buteyko breathing technique in asthma as taught by a video. J Asthma 2000;37:557-64.

6 Ware JE, Sherbourne CD. The MOS 36-item short-form health survey (SF-36). I. Conceptual framework and item selection. Med Care 1992;30:473-83.

7 Juniper EF, Guyatt GH, Ferrie PJ, et al. Measuring quality of life in asthma. Am Rev Respir Dis 1993;147:832-8.

8 Egbagbe E, Pavord ID, Wilding P, et al. Adenosine monophosphate and histamine induced bronchoconstriction: repeatability and protection by terbutaline. Thorax 1997;52:239-43.

9 Buteyko Breathing Centre. http://www.buteyko.co.uk/trials.html

10 Hensley MJ, Gibson PG. Promoting evidence-based alternative medicine. Med J Aust 1998;169:573-4.

11 Thomas M, McKinley, RK, Freeman E, et al. Prevalence of dysfunctional breathing in patients treated for asthma in primary care: cross sectional survey. BM 2001;322:1098-100.

12 Keeley D, Osman L. Dysfunctional breathing and asthma. BM 2001;322:1075-6.

13 Harrison TW, Oborne J, Wilding PJ, et al. Randomised placebo controlled trial of beta-agonist reduction in asthma. Thorax 1999:54:98-102.

14 Manocha R, Marks GB, Kenchington P, et al. Sahaja yoga in the management of moderate to severe asthma: a randomised controlled trial. Thorax 2002;57: 110-5.

15 McCarthy M. US panel calls for more support of alternative medicine. Lancet 2002;359:1213.

16 House of Lords Select Committee on Science and Technology. Complementary and alternative medicine. 6th report 1999-2000 [HL123]. London: Stationery Office, 2000.

\section{LUNG ALERT}

\section{Anti-CD44 antibodies decrease airway inflammation and} hyperresponsiveness in a murine model of allergic disease

$\Delta$ Katoh S, Matsumoto N, Kawakita K, et al. A role for CD44 in an antigen-induced murine model of pulmonary eosinophilia. J Clin Invest 2003;111:1563-70

$\square$ his study investigates the effects of anti-CD44 murine antibodies on allergic airway inflammation induced by transnasal instillation of purified Ascaris suum (Asc) antigen.

Two antibodies were used-one that directly blocks hyaluronic acid binding to cells expressing CD44, and one that causes CD44 receptor to be shed from tissue surfaces.

The study showed that the total number of airway eosinophils and lymphocytes increased after challenge with Asc and both types of anti-CD44 antibody significantly suppressed cell levels $(p<0.05)$. Asc induced increases in interleukin (IL) - 4 and IL-5 levels in bronchoalveolar lavage fluid were reduced after use of anti-CD44 antibodies $(p<0.05)$. Levels of eotaxin and TARC were also reduced $(\mathrm{p}<0.05)$. Airway hyperresponsiveness to methacholine was analysed; anti-CD44 antibodies significantly reduced the response in Asc treated mice compared with rat IgG $(\mathrm{p}<0.05)$. Hyperresponsiveness was also significantly decreased in mite (Dermatophagoides) allergen treated mice given anti-CD44 antibody $(\mathrm{p}<0.05)$

These results show that CD44 may play an important role in allergic airway inflammation through its actions on leucocyte binding and stimulation of cytokine production. Further evaluation of the interactions between IL-4, IL-5, and CD44 may determine which factor is most influential in airway inflammation and clarify the role of the eosinophil in asthma.

K Ryanno 